\title{
A Nash Bargaining Solution for Cooperative Network Formation Games
}

\author{
Konstantin Avrachenkov ${ }^{1}$, Jocelyne Elias ${ }^{2}$, Fabio Martignon ${ }^{3}$, \\ Giovanni Neglia ${ }^{1}$, and Leon Petrosyan ${ }^{4}$ \\ 1 INRIA Sophia Antipolis \\ \{Konstantin. Avrachenkov, Giovanni .Neglia\}@sophia.inria.fr \\ 2 Paris Descartes University \\ jocelyne.elias@parisdescartes.fr \\ 3 University of Bergamo \\ fabio.martignon@unibg. it \\ 4 St. Petersburg State University \\ lapetr@apmath.spbu.ru
}

\begin{abstract}
The Network Formation problem has received increasing attention in recent years. Previous works have addressed this problem considering almost exclusively networks designed by selfish users, which can be consistently suboptimal.

This paper addresses the network formation issue using cooperative game theory, which permits to study ways to enforce and sustain cooperation among agents. Both the Nash bargaining solution and the Shapley value are widely applicable concepts for solving these games. However, we show that the Shapley value presents three main drawbacks in this context: (1) it is non-trivial to define meaningful characteristic functions for the cooperative network formation game, (2) it can determine for some players cost allocations that are even higher than those at the Nash Equilibrium (i.e., if players refuse to cooperate), and (3) it is computationally very cumbersome.

For this reason, we solve the cooperative network formation game using the Nash bargaining solution (NBS) concept. More specifically, we extend the NBS approach to the case of multiple players and give an explicit expression for users' cost allocations. Furthermore, we compare the NBS to the Shapley value and the Nash equilibrium solution, showing its advantages and appealing properties in terms of cost allocation to users and computation time to get the solution.

Numerical results demonstrate that the proposed Nash bargaining solution approach permits to allocate costs fairly to users in a reasonable computation time, thus representing a very effective framework for the design of efficient and stable networks.
\end{abstract}

Index Terms: Network Formation, Cooperative Game Theory, Coalition, Nash bargaining solution, Shapley value.

\section{Introduction}

The Network Formation problem has become increasingly important given the continued growth of computer networks such as the Internet. The design of such

J. Domingo-Pascual et al. (Eds.): NETWORKING 2011, Part I, LNCS 6640, pp. 307-318, 2011.

(C) IFIP International Federation for Information Processing 2011 
networks is generally carried out by a large number of self-interested actors (users, Internet Service Providers ... ), all of whom seek to optimize the quality and cost of their own operation.

Over the past years, the network formation problem has been tackled almost exclusively from a non-cooperative point of view. Recent works 1 1|2/3445 have modeled how independent selfish agents can build or maintain a large network by paying for possible edges. Nash equilibria in such games, however, can be much more expensive than the optimal, centralized solution. This is mainly due to the lack of cooperation among network users, which leads to design costly networks.

The underlying assumption in all the above works is that agents are completely non-cooperative, isolated entities. However, this assumption could be not entirely realistic, for example when network design involves long-term decisions (e.g., in the case of Autonomous Systems peering relations). It is more natural that agents will discuss possible strategies and, as in other economic markets, form coalitions taking strategic actions that are beneficial to all members of the group. Moreover, incentives could be introduced by some external authority (e.g., the network administrator, government authority) in order to increase the users' cooperation level.

Preliminary works, like [6]7, tried to overcome this limitation by incorporating a socially-aware component in the users' utility functions. This solution, though, can be insufficient to obtain cost-efficient networks in all scenarios. In fact, it has been demonstrated in [6] that, quite surprisingly, highly socially-aware users can form stable networks that are much more expensive than the networks designed by purely selfish users.

To address the above issues, in this paper we formulate the network formation problem as a cooperative game, where groups of players (named coalitions) coordinate their actions and pool their winnings; consequently, one of the problems is how to divide the cost savings among the members of the formed coalition.

The Shapley value and the Nash bargaining solution are widely applicable solution concepts for cooperative games. The former has appealing properties, since it provides a unique and fair solution [8. The Nash bargaining approach, on the other hand, studies situations where two or more agents need to select one of the many possible outcomes of a joint collaboration 910. Examples include wage negotiation between an employer and a potential employee, or trade negotiation between two countries. Each party in the negotiation has the option of leaving the table, in which case the bargaining will result in a disagreement outcome. The Nash bargaining solution (NBS) is a very effective tool to model interactions among negotiators, and is unique for bargaining games satisfying Pareto optimality, symmetry, scale independence, and independence of irrelevant alternatives 910.

However, we will show that the Shapley value presents several drawbacks in this context: (1) it is non-trivial to define meaningful characteristic functions for the cooperative network formation game, (2) the cost allocation determined by the Shapley value can be, in some cases, even costlier than that obtained at 
some Nash equilibrium, and (3) for our network formation game, it cannot be determined in a reasonable computation time.

For these reasons, we propose a Nash bargaining approach to solve the cooperative network formation problem. More specifically, as a key contribution, we extend the Nash bargaining solution for the cooperative network formation problem to the case of multiple players with linear constraint, and give explicit expressions for users' cost allocations. To the best of our knowledge, the derived explicit expressions are new.

Furthermore, we perform a thorough comparison of the proposed Nash bargaining solution with other classic approaches like the Shapley value and the Nash equilibrium solutions, using different network scenarios.

Numerical results demonstrate that the proposed Nash bargaining solution can compute efficient cost allocations in a short computing time, thus representing a very effective tool to plan efficient and stable networks.

The main contributions of this work can therefore be summarized as follows:

- the formulation of the network formation problem as a cooperative game, where players cooperate to reduce their costs.

- The proposition of a novel Nash bargaining solution for the $n$-person cooperative network formation problem, which has appealing properties in terms of planning efficient networks and cost allocations in a reasonable computation time.

- A comparison of the proposed approach with classic solutions, like the Shapley value and the Nash equilibrium concepts, in large-size network topologies.

The paper is organized as follows: Section 2 discusses related work. Section 3 introduces the cooperative network formation game, the proposed Nash bargaining solution. Section 4 presents numerical results that demonstrate the effectiveness of the NBS approach in different realistic network scenarios. Finally, Section 5 concludes this paper.

\section{Related Work}

The network formation problem has been addressed in several recent works, mainly in the context of non-cooperative games [126]. The works in 341112 have further considered coordination issues among players.

The so-called Shapley network design game is proposed in [1]. In this noncooperative network formation game, each player chooses a path from its source to its destination, and the overall network cost is shared among the players in the following way: each player pays for each edge a proportional share $\frac{c_{e}}{x_{e}}$ of the edge $\operatorname{cost} c_{e}$, where $x_{e}$ is the number of players that choose such edge. In [6], the Shapley network design game is extended, adding a socially-aware component to users' utility functions.

The survey article in [11] presents the most notable works on network formation in cooperative games; furthermore, the existence of networks that are stable against changes in link choices by any coalition is studied in [13. In [14, 
Andelman et al. analyze strong equilibria with respect to players' scheduling as well as a different class of network creation games in which links may be formed between any pair of agents. For these latter games, strong Nash equilibria (i.e., equilibria where no coalition can improve the cost of each of its members) achieve a constant Price of Anarchy, which is defined as the ratio between the cost of the worst Nash equilibrium and the social optimum. Strong Nash equilibria ensure stability against deviations by every conceivable coalition of agents. A similar problem is considered in [12, where nodes can collaborate and share the cost of creating any edge in the host graph.

The works in [3] study the existence of strong Nash equilibria in network design games under different cost sharing mechanisms. More specifically, the authors in [3] show that there are graphs that do not admit strong Nash equilibria, and then give sufficient conditions for the existence of approximate strong Nash equilibria.

The idea of using the Nash bargaining solution in the context of telecommunication networks has been considered in different networking scenarios. Such approach was first presented for packet-switched data networks by Mazumdar et al. [15. The concept of Nash bargaining solution is used by Yaiche et al. [16] to derive a price-based resource allocation scheme that can be applied to the available bit rate service in ATM networks. In [17] the authors propose a scheme to allocate subcarrier, rate, and power for multiuser orthogonal frequency-division multipleaccess systems. The approach considers a fairness criterion, which is a generalized proportional fairness based on Nash bargaining solutions and coalitions.

The reader is referred to the next section, to the book by Muthoo [9] and the paper by Nash 10, for a general introduction to the Nash bargaining solution concept.

\section{Cooperative Network Formation Game: Formulation and Solutions}

This section illustrates the cooperative network formation game considered in this work, and describes the proposed Nash bargaining solution (NBS). A review of the Shapley value approach is preliminarily proposed for comparison reasons.

\subsection{Network Model}

We are given a directed graph $G=(V, E)$, where each edge $e$ has a nonnegative $\operatorname{cost} c_{e}$; each player $i \in \mathcal{I}=\{1,2, \ldots n\}$ is identified with a source-destination pair $\left(s_{i}, t_{i}\right)$, and wants to connect his source to the destination node with the minimum possible cost. Note that $c_{e}$ represents the total edge cost, which is shared among the players according to the allocation algorithms we will describe in the following.

We consider a cooperative game in strategic form $G=\left\langle\mathcal{I}, A,\left\{J^{i}\right\}\right\rangle$, where $\mathcal{I}$ is the set of players, $A_{i}$ is the set of actions for player $i, A=A_{1} \times \ldots \times A_{n}$, and $J^{i}$ is the objective (cost) function, which player $i$ wishes to optimize (minimize). 
In a cooperative game, players bargain with each other before the game is played. If an agreement is reached, players act according to such agreement, otherwise players act in a non-cooperative or antagonistic way. Note that the agreements must be binding, so players are not allowed to deviate from what is agreed upon.

\subsection{The Shapley Value Solution}

We now review the Shapley value solution approach, and discuss meaningful definitions for the characteristic function.

The Shapley value is a widely applied concept for solving cooperative games. It is a possible way to allocate the total costs among the members of a coalition, taking into account their different importance for the coalition. The main advantage of the Shapley value is that it provides a solution that is both unique and fair: it is unique in the class of subadditive cooperative games (see definition below); it is fair in a sense that it satisfies a series of axioms intuitively associated with fairness (see [8]). However, while these are both desirable properties, the Shapley value has one major drawback: for many coalition games, including our network formation game, it cannot be determined in a reasonable time. We shall discuss computation aspects in more detail below.

A Shapley function $\phi$ is a function that assigns to each possible characteristic function $v$ a vector of real numbers, i.e.,

$$
\phi(v)=\left[\phi_{1}(v), \ldots, \phi_{i}(v), \ldots, \phi_{n}(v)\right]
$$

where $\phi_{i}(v)$ represents the cost of player $i$ in the game.

The characteristic function, $v$, is a real-valued function that associates with every non-empty subset $\mathcal{S}$ of $\mathcal{I}$ (i.e., a coalition) a real number $v(\mathcal{S})$, the cost of $\mathcal{S} ; v(\mathcal{S})$ must satisfy the following properties:

1. $v(\emptyset)=0$.

2. (Subadditivity) if $\mathcal{S}$ and $\mathcal{T}$ are disjoint coalitions $(\mathcal{S} \cap \mathcal{T}=\emptyset)$, then $v(\mathcal{S})+$ $v(\mathcal{T}) \geq v(\mathcal{S} \cup \mathcal{T})$

This latter property means that cooperation can only help but never hurt.

Note that defining the characteristic function is not straightforward for the cooperative network formation game considered in this work, since a "natural" definition can violate the subadditivity property, as we will discuss in the following.

The three definitions reported hereafter "naturally" arise in our networking problem as candidate characteristic functions:

1. Players in $\mathcal{S}$ and players in $\mathcal{I}-\mathcal{S}$ form two separate coalitions. Each coalition tries to minimize the total cost for its members, taking into account the selfish behavior of the other coalition. A Nash equilibrium is reached, and $v(\mathcal{S})$ is defined as the total cost for members in $\mathcal{S}$ at this equilibrium.

$\overline{1}$ The second one is required to guarantee the uniqueness of the Shapley value solution. 
2. The value of the coalition $\mathcal{S}$ is defined as its security level, i.e. as the minimum total cost that $\mathcal{S}$ can guarantee to itself when members in $\mathcal{I}-\mathcal{S}$ act collectively in order to maximize the cost for the coalition $\mathcal{S}$.

3. The value of coalition $\mathcal{S}$ is equal to the minimum cost that its members would incur if players in $\mathcal{I}-\mathcal{S}$ would be absent.

We note that, in our specific game, these three definitions give increasing value to a coalition $\mathcal{S}$. In fact, when players in $\mathcal{I}-\mathcal{S}$ minimize their own cost (first definition), their path choices cannot be as bad for $\mathcal{S}$ as when they try to maximize the cost for $\mathcal{S}$ (second definition). Still, when players in $\mathcal{I}-\mathcal{S}$ are present, they are obliged to select paths to connect their source-destination pairs, and some of these links may be used also by players in $\mathcal{S}$, so that $v(\mathcal{S})$ is smaller in the second definition than in the third.

To better illustrate the differences underlying these definitions, let us consider the hexagon network scenario of Figure 1 with 6 links and 3 players having the following source-destination pairs: $\left(s_{1}, t_{1}\right),\left(s_{2}, t_{2}\right)$ and $\left(s_{3}, t_{3}\right)$. All link costs are equal to 1 , except for link $t_{3} \rightarrow t_{2}$, which has a cost equal to $1-\epsilon, \epsilon$ being a very small constant.

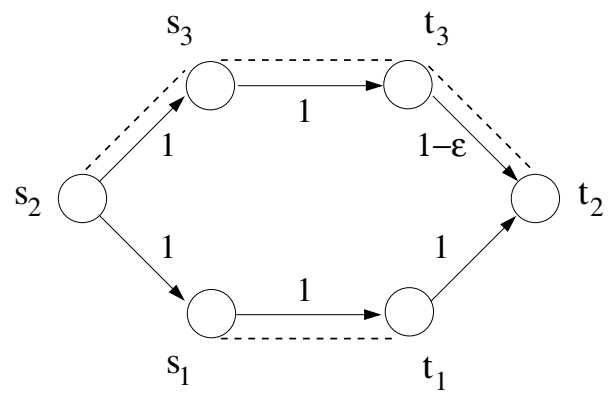

Fig. 1. Hexagon network topology: the 3 players must connect their source-destination nodes $\left(s_{i}, t_{i}\right)$. The optimal solution, which in this case coincides with both the Nash equilibrium point and the Nash bargaining solution, is illustrated with dashed lines.

Table 1 reports, for each of the three above definitions, the corresponding characteristic function values. It can be easily checked that definition (1) does not lead to a characteristic function, since the subadditivity property is not satisfied (for example, $v(12)+v(3)<v(123)$ ), and therefore it cannot be used to compute Shapley values. Indeed, with such definition, cooperation among players can lead to costlier solutions. On the other hand, definitions (2) and (3) lead to characteristic functions.

Theorem 1. In the Cooperative Network Formation Game, the security level (definition 2) and the minimum cost of the coalition (definition 3) satisfy the axioms of characteristic function.

Proof: See Research Report [18]. 
Table 1. Hexagon network scenario: characteristic function values, $v(\mathcal{S})$, for definitions (1), (2) and (3)

\begin{tabular}{|c|c|c|c|}
\hline & \multicolumn{3}{|c|}{ Characteristic Function value $(v(\mathcal{S}))$} \\
\hline Coalition $(\mathcal{S})$ & Definition (1) & Definition $(2)$ & Definition $(3)$ \\
\hline$\emptyset$ & 0 & 0 & 0 \\
\hline 1 & 1 & 1 & 1 \\
\hline 2 & $2.5-\epsilon$ & $2.5-\epsilon$ & $3-\epsilon$ \\
\hline 3 & 0.5 & 1 & 1 \\
\hline 12 & 3 & 3 & 3 \\
\hline 13 & 1.5 & 1.5 & 2 \\
\hline 23 & $3-\epsilon$ & $3-\epsilon$ & $3-\epsilon$ \\
\hline 123 & $4-\epsilon$ & $4-\epsilon$ & $4-\epsilon$ \\
\hline
\end{tabular}

To calculate the Shapley function, suppose we form the grand coalition (the coalition containing all $n$ players) by entering the players into this coalition one at a time. As each player enters the coalition, he is charged the cost by which his entry increases the cost of the coalition he has entered. The cost a player pays by this scheme depends on the order in which the players enter. The Shapley value is just the average cost charged to the players if they enter in a completely random order, i.e.

$$
\phi_{i}=\sum_{\mathcal{S} \subset \mathcal{I}, i \in \mathcal{S}} \frac{(|\mathcal{S}|-1) !(n-|\mathcal{S}|) !}{n !}(v(\mathcal{S})-v(\mathcal{S}-\{i\})) .
$$

It can be proved that the problem of computing the Shapley value is an NPcomplete problem. Polynomial methods, based on sampling theory, have been proposed in [19] for approximating the Shapley value; these estimations, though, are efficient only if the worth of any coalition $\mathcal{S}$ can be calculated in polynomial time, which is not the case for our problem.

In fact, even using the approximation methods proposed for example in [19], it is necessary to compute the worth of an extremely large number of coalitions, which is computationally very cumbersome, while as we see next, our proposed Nash bargaining solution needs only computing the worth of the grand coalition.

\subsection{The Nash Bargaining Solution (NBS)}

Since the computation cost of the Shapley value can be extremely high in network scenarios with many players, in this paper we consider another approach to cooperative game: Nash bargaining. We will show that the computation of the Nash bargaining solution is very light.

Let $u_{i}$ denote the maximal acceptable cost that user $i$ is willing to pay. In the present work we suggest the following three options:

1. the cost for user $i$ to connect its source-destination nodes in a purely noncooperative game (i.e., the Nash equilibrium solution); 
2. the cost for user $i$ to connect its source-destination nodes in a zero-sum game where all the other players are trying to maximize the cost of user $i$;

3 . the cost for user $i$ to connect its source-destination nodes when there is no other player.

The vector $u=\left\{u_{1}, u_{2}, \ldots u_{n}\right\}$ is also denoted as the disagreement point of the cooperative network formation game (i.e., what will happen if players cannot come to an agreement). Clearly, the cost achieved by every player at any agreement point (every possible outcome of the bargaining game) has to be at most equal to the cost achieved at the disagreement point.

We now derive a Nash bargaining solution for allocating the total network cost to users. To this aim, we extend the well-known two-player NBS concept to the $n$-player network formation game, considering transferable network costs, providing explicit expressions. This assumption means that the players or the system administrator can redistribute the total cost among the players.

Let $u_{s o c}$ denote the total network cost resulting from social optimization. This can be computed, for example, formulating the generalized Steiner Tree problem 20] with an Integer Linear Program, using a mathematical programming model (like AMPL), and solving it with a commercial solver (like CPLEX). Solving such problem provides the least-cost network topology that connects all source-destination pairs.

Then, the Nash bargaining solution can be given in explicit form.

Theorem 2. The Nash bargaining solution for player $i, \alpha_{i}$ is given by the following expression:

$$
\alpha_{i}=u_{i}-\frac{\sum_{k} u_{k}-u_{s o c}}{m}
$$

where $m$ coincides with the number of players $n$ (i.e., $m \equiv n$ ) if we allow for negative costs (i.e., some $\alpha_{i}$ values are negative, which means that some players are actually paid to ensure their participation). Otherwise, if only non-negative costs are allowed (or equivalently, if no positive transfers are permitted), $m$ is defined as the largest integer for which the following inequality is satisfied:

$$
\frac{1}{m-1}\left(\sum_{i=1}^{m-1} u_{i}-u_{s o c}\right)<u_{m}
$$

having assumed, without loss of generality, that players are ordered such that $u_{1} \geq u_{2} \geq \ldots \geq u_{n}$.

Proof: See Research Report [18].

We would like to emphasize that in the first case $\alpha$ values can be positive or negative, while in the second case $\alpha$ values are non-negative. In particular, $m$ gives the number of non-zero $\alpha$ values, i.e., $\alpha_{1}, \alpha_{2}, \ldots \alpha_{m}$ are positive and given by expression (3), while $\alpha_{m+1}, \ldots \alpha_{n}$ are equal to zero. 


\section{Numerical Results}

This section reports the numerical results obtained applying our proposed Nash bargaining solution (NBS) to cooperative network design games played in various network scenarios, including simple network instances and more complex random topologies. The NBS, computed as illustrated in the previous section, is compared both to the cost allocation provided by the Shapley value, as well as to a Nash equilibrium solution. This latter is determined in the non-cooperative network formation framework proposed in [1, revised in Section 2, starting from the empty network and using a best response algorithm where each user greedily minimizes its path cost until an equilibrium is reached.

We assume that positive transfers are allowed. To compute the Shapley value, we further assume that the worth of a coalition $\mathcal{S}$ is the minimum cost that its members would incur if players in $\mathcal{I}-\mathcal{S}$ would be absent (definition 3 ). This allows us to consider the "worst case", i.e. the costlier definition for a coalition, as discussed before. As for the disagreement point $u_{i}$ in the NBS, we reasonably assume that it is the cost for user $i$ to connect its source-destination nodes in a purely non-cooperative game (i.e., the Nash equilibrium solution). However, we underline that our proposed NBS approach is general and can be applied to any problem setting. The investigation of the impact of other characteristic function and disagreement point choices is left as future research issue.

Let us first consider the simple network scenario already illustrated in Figure1, with 6 links and 3 players. The optimal network cost is here $u_{\text {soc }}=4-\epsilon$, and coincides with the cost of the network formed at the Nash Equilibrium Point (NEP). The Nash equilibrium and the Shapley value solutions for this scenario are reported in Table 2, together with the Nash bargaining solution, which in this case coincides with the NEP.

Numerical results show that the solution given by the Shapley value for player $3\left(\frac{5-2 \epsilon}{6} \approx 0.83\right)$ is costlier than that of the Nash equilibrium, 0.5 . We further observe that even defining the value of a coalition as its security level (definition 2, Section 3.2) leads to the same Shapley values reported in Table 2. As a consequence, the Shapley value solution is somehow unstable for all the considered definitions of the characteristic function, since some players (i.e., player 3 in this scenario) can deviate to reduce their cost. This is surprising, because the Shapley value satisfies the individual rationality property, so that the Shapley value allocation is always preferable for each player than playing alone. The

Table 2. Hexagon network scenario with 3 players. The table reports the cost paid by each player at the Nash Equilibrium Point, the Shapley value and the Nash bargaining solution. The total network cost is equal to $4-\epsilon$ for all allocation algorithms.

\begin{tabular}{|c|c|c|c|}
\hline Algorithm & $\left(s_{1}, t_{1}\right)$ & $\left(s_{2}, t_{2}\right)$ & $\left(s_{3}, t_{3}\right)$ \\
\hline NEP & 1 & $2.5-\epsilon$ & 0.5 \\
\hline Shapley value & $\frac{5+\epsilon}{6} \approx 0.83$ & $\frac{14-5 \epsilon}{6} \approx 2.33$ & $\frac{5-2 \epsilon}{6} \approx 0.83$ \\
\hline NBS & 1 & $2.5-\epsilon$ & 0.5 \\
\hline
\end{tabular}


apparent paradox originates from the fact that the value of the single player coalition has been defined either as the cost incurred if all other players are absent (definition 3), or as its security value, considering that all the other players are trying to maximize its cost (definition 2). In reality, at the Nash equilibrium, the cost of player $i$ is smaller than such values and, as we have shown, it can be even smaller than the Shapley value imputation.

The same behavior can be observed also in more general topologies. To show this, we considered random network scenarios generated as follows: we randomly extract the position of $N$ nodes, uniformly distributed on a square area with edge equal to 1000. As for the network links, which can be bought by players to connect their endpoints, we consider random geometric graphs, where links exist between any two nodes located within a range $R$. The link cost is set to its length.

Table 3 and Figure 2 illustrate the results obtained in a random geometric graph scenario with 50 nodes, range $R=500$ (which means approximately more than 1200 links) and, respectively, 10 and 15 source-destination pairs (players). The table and figure report the costs for the players reached at the Nash equilibrium, the Shapley value as well as our proposed Nash bargaining solution. The total network cost is reported in the last column; note that such value corresponds, for the Shapley value and the Nash bargaining allocation algorithms, to the socially optimal solution $\left(u_{\text {soc }}\right.$ parameter), which can be obtained as explained in Section 3.)

It can be observed that, in all scenarios, at least 2 players (marked in bold in the table, with arrows in Figure 2) have a Shapley value that is higher than the Nash equilibrium cost. However, the cost saving between the NEP and the optimal cost (which is approximately 700 and 1250 for the $n=10$ and $n=15$ scenarios, respectively) could be re-distributed, which is what the Nash bargaining solution does, increasing the appeal of the cost sharing solution.

Obviously, since both the Shapley value and the NBS distribute the social cost $\left(u_{s o c}\right)$ among the players, there will be players whose allocation is costlier under the NBS than with the Shapley value allocation. This happens, in the numerical examples we considered, for players that have a large cost at the Nash equilibrium. However, every player is always better off under the NBS allocation than at the Nash equilibrium, since cost savings are redistributed.

Furthermore, we observe that computing the Shapley value for $n=15$ players took several weeks of computation on the workstation used to obtain the numerical results reported in this paper, i.e., an Intel Pentium 4 (TM) processor with

Table 3. Random geometric network scenario with 10 players. The table reports the cost paid by each player at the Nash Equilibrium Point, the Shapley value and the Nash bargaining solution. The total network cost is also reported.

\begin{tabular}{|c|c|c|c|c|c|c|c|c|c|c|c|}
\hline Algorithm & P1 & P2 & P3 & P4 & P5 & P6 & P7 & P8 & P9 & P10 & Total cost \\
\hline NEP & 283.0 & 149.4 & 235.3 & 824.4 & 714.8 & 450.5 & 674.0 & 195.6 & 186.0 & 266.9 & 3979.9 \\
\hline Shapley value & 260.5 & $\mathbf{1 7 0 . 6}$ & $\mathbf{2 5 3 . 9}$ & 717.1 & 472.8 & 387.5 & 508.0 & 142.5 & 183.8 & 175.6 & 3272.2 \\
\hline NBS & 212.3 & 78.6 & 164.6 & 753.6 & 644.0 & 379.7 & 603.2 & 124.8 & 115.3 & 196.2 & 3272.2 \\
\hline
\end{tabular}




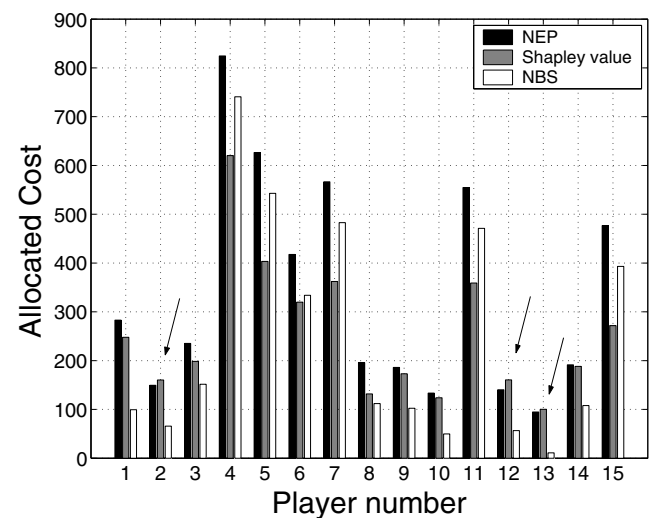

Fig. 2. Random geometric network scenario with 15 players. The figure reports the cost paid by each player at the NEP, the Shapley value and the NBS. The total network cost is equal to 5076.0 at the NEP and to 3802.7 for the Shapley value and NBS allocations.

CPUs operating at $3 \mathrm{GHz}$ and with 1024 Mbyte of RAM. Therefore, computing the Shapley value for a larger number of players is practically infeasible in such network scenario. On the other hand, our proposed $n$-person Nash bargaining solution is very simple to calculate, and could be computed within a few minutes in all considered network scenarios, thus representing a practical and efficient solution to the network formation problem.

\section{Conclusion}

In this paper we proposed a novel and efficient Nash bargaining solution for the cooperative network formation problem with $n$ players. Our solution has very appealing properties in terms of planning efficient networks and determining cost allocations in a very short computation time.

We compared our proposed solution to classic approaches, like the Shapley value and the Nash equilibrium concepts, in simple and large-size network topologies, with an increasing number of players.

Numerical results demonstrate that our approach permits to achieve very effective cost allocations, thus representing an efficient an promising framework for the planning of stable networks.

\section{References}

1. Anshelevich, E., Dasgupta, A., Kleinberg, J., Tardos, E., Wexler, T., Roughgarden, T.: The price of stability for network design with fair cost allocation. In: Proc. of the 45th Annual Symposium on Foundations of Computer Science (FOCS), Rome, Italy, October 17-19, pp. 295-304 (2004)

2. Chen, H.L., Roughgarden, T.: Network design with weighted players. In: Proc. of the 18th ACM Symposium on Parallelism in Algorithms and Architectures (SPAA 2006), Cambridge, MA, USA, July 30-August 2 (2006) 
3. Albers, S.: On the value of coordination in network design. In: Proc. of the 19th Annual ACM-SIAM Symposium on Discrete Algorithms (SODA), San Francisco, CA, USA, pp. 294-303 (2008)

4. Epstein, A., Feldman, M., Mansour, Y.: Strong equilibrium in cost sharing connection games. In: Proc. of the 8th ACM Conference on Electronic Commerce, San Diego, CA, USA, pp. 84-92 (June 2007)

5. Chen, H.L., Roughgarden, T., Valiant, G.: Designing networks with good equilibria. In: Proc. of the 19th Annual ACM-SIAM Symposium on Discrete Algorithms, pp. 854-863 (2008)

6. Elias, J., Martignon, F., Avrachenkov, K., Neglia, G.: Socially-Aware Network Design Games. In: Proc. of INFOCOM 2010, San Diego, CA, USA (2010)

7. Azad, A.P., Altman, E., ElAzouzi, R.: From Altruism to Non-Cooperation in Routing Games. In: Proc. of Networking and Electronic Commerce Research Conference, Lake Garda, Italy (October 2009)

8. Aumann, R.J., Myerson, R.B., Roth, A.: The Shapley Value. Game-Theoretic Methods in General Equilibrium Analysis, 61-66 (1994)

9. Muthoo, A.: Bargaining theory with applications. Cambridge Univ. Press, Cambridge (1999)

10. Nash Jr., J.F.: The bargaining problem. Econometrica 18(2), 155-162 (1950)

11. van den Nouweland, A.: Models of network formation in cooperative games. Cambridge Univ. Press, Cambridge (2005)

12. Demaine, E.D., Hajiaghayi, M., Mahini, H., Zadimoghaddam, M.: The Price of Anarchy in Cooperative Network Creation Games. In: Proc. of STACS 2009, Freiburg, Germany, February 26-28, pp. 301-312 (2009)

13. Jackson, M., van den Nouweland, A.: Strongly stable networks. Games and Economic Behavior 51, 420-444 (2005)

14. Andelman, N., Feldman, M., Mansour, Y.: Strong price of anarchy. In: Proceedings of the 18th Annual ACM-SIAM Symposium on Discrete Algorithms (SODA 2007), New Orleans, Louisiana, January 7-9 (2007)

15. Mazumdar, R., Mason, L.G., Douligeris, C.: Fairness in network optimal flow control: Optimality of product forms. IEEE Trans. Comm. 39(5), 775-782 (1991)

16. Yaiche, H., Mazumdar, R.R., Rosenberg, C.: A game theoretic framework for bandwidth allocation and pricing in broadband networks. IEEE/ACM Transactions on Networking 8(5), 667-678 (2000)

17. Han, Z., Ji, Z., Ray Liu, K.J.: Fair Multiuser Channel Allocation for OFDMA Networks Using Nash Bargaining Solutions and Coalitions. IEEE Trans. Comm. 53(8), 1366-1376 (2005)

18. Avrachenkov, K., Elias, J., Martignon, F., Neglia, G., Petrosyan, L.: A Nash bargaining solution for Cooperative Network Formation Games. In: INRIA Research Report no. 7480 (December 2010), http://hal.archives-ouvertes.fr/inria-00544527/en/

19. Castro, J., Gomez, D., Tejada, J.: Polynomial calculation of the Shapley value based on sampling. Computers \& Operations Research 36(5) (2009)

20. Khan, M., Kuhn, F., Malkhi, D., Pandurangan, G., Talwar, K.: Efficient distributed approximation algorithms via probabilistic tree embeddings. In: Proc. of the 27th Symposium on Principles of Distributed Computing, pp. 263-272 (2008) 